\title{
PYRUVATE PARTICIPATION IN THE LOW MOLECULAR WEIGHT TROPHIC ACTIVITY FOR CENTRAL NERVOUS SYSTEM NEURONS IN GLIA-CONDITIONED MEDIA ${ }^{1}$
}

\author{
IVAN SELAK, ${ }^{2}$ STEPHEN D. SKAPER, ${ }^{3}$ AND SILVIO VARON \\ Department of Biology, School of Medicine, University of California, San Diego, La Jolla, California 92093
}

Received April 23, 1984; Revised June 20, 1984; Accepted June 22, 1984

\begin{abstract}
Conditioned media from glial cell cultures contain low molecular weight agents which can support survival of CNS neurons in the absence of recognized protein neuronotrophic factors. A similar support is provided to CNS neurons by selected basal media, and pyruvate is the critical medium constituent responsible for their trophic competence. Eagle's basal medium, which contains no pyruvate, acquires pyruvate when conditioned over astroglial cell cultures. Enzymatic degradation of the pyruvate in the astroglia-conditioned medium leads to corresponding losses in its low molecular weight trophic activity for CNS neurons. Quantitative correlations between pyruvate content and CNS trophic activity demonstrate that $(i)$ pyruvate is the main trophic ingredient of the glia-conditioned medium, and (ii) other low molecular weight substances, acquired during conditioning, reduce the pyruvate concentration required for its trophic effect. The "pyruvate-sparing" substances, as yet unidentified, are not the serine and $\mathrm{Fe}^{3+}$ which have pyruvate-sparing competence for peripheral, ciliary ganglionic neurons. These findings, together with previous observations, propose that prenatal neurons fail to generate or retain endogenous pyruvate at the levels for their survival-sustaining activities.
\end{abstract}

Neuronotrophic factors are special proteins required for the survival of selected neuronal populations (cf. Varon and Adler, 1981; Varon et al., 1984a). Recent studies have revealed that neurons from the CNS can be maintained in dissociated cell cultures without the addition of exogenous neuronotrophic factors if supplied with a low molecular weight (LMW) fraction from glia and other conditioned media (Manthorpe et al., 1982; Varon et al., 1984c). The chemical nature of these LMW $(<1000)$ trophic agents has not been identified.

In another, independent study (Skaper et al., 1984), we have established that exogenous pyruvate is essential for the survival of at least two-thirds of the neurons dissociated from 8-day chick embryo ciliary ganglia (CG8). These ciliary neurons also require their macromolecular ciliary neuronotrophic factor (CNTF), and the insulin component of the serum-replacing N1 supplement (a mixture of insulin, transferrin, progesterone, putrescine, and selenite). Half of the pyruvate-dependent CG8 neurons also require serine plus $\mathrm{Fe}^{3+}$. For all the pyruvaterequiring CG8 neurons, the concurrent availability of exogenous serine and $\mathrm{Fe}^{3+}$ reduces by 50 -fold the pyruvate concentration needed. The present report extends those studies to several populations of prenatal, intrinsic CNS neurons (both rodent and avian).

\section{Materials and Methods}

Culture media and supplements. The following media were utilized: Eagle's basal medium (EBM), Dulbecco's modified Eagle's medium

\footnotetext{
${ }^{1}$ This work was supported by a grant from FIDIA Research Laboratories.

${ }^{2}$ Aspirant du FNRS. Current address: Department de Clinique et de Pathologie Medicales, University of Liége (Belgium).

${ }^{3}$ To whom correspondence should be addressed.
}

(DMEM), Ham's F-12, Leibowitz L-15, RPMI-1640, and Eagle's minimal essential medium (MEM). All media were supplemented with 26.4 $\mathrm{mM} \mathrm{NaHCO}, 2 \mathrm{mM}$ L-glutamine, and 100 units $/ \mathrm{ml}$ of penicillin. DGlucose, when present, was at 5.6 or $33.3 \mathrm{~mm}$. 'The serum-replacing supplement consists of insulin $\left(8.3 \times 10^{-7} \mathrm{M}\right)$, transferrin $\left(6.2 \times 10^{-8}\right.$ $\mathrm{M})$, putrescine $\left(1.0 \times 10^{-4} \mathrm{M}\right)$, progesterone $\left(2.0 \times 10^{-8} \mathrm{M}\right)$, and sodium selenite $\left(3.0 \times 10^{-8} \mathrm{M}\right)$ (Skaper et al., 1979; Bottenstein et al., 1980). In some instances, the N1-supplemented basal media also received sodium pyruvate $\left(1.0 \times 10^{-3} \mathrm{M}\right)$, glycine $\left(4.0 \times 10^{-4} \mathrm{M}\right)$, serine $(4.0 \times$ $\left.10^{-4} \mathrm{M}\right)$, and $\mathrm{FeCl}_{3}\left(2.5 \times 10^{-7} \mathrm{M}\right)$. Astroglial conditioned medium (ACM) was obtained from confluent cultures of primary rat brain astroglial cells, incubated at $37^{\circ} \mathrm{C}$ in EBM for $48 \mathrm{hr}$ (Manthorpe et al., 1982). A LMW fraction was obtained from ACM by subjecting the latter to ultrafiltration through a PM-10 membrane with a 10,000 dalton cutoff (Varon et al., 1984c).

Neuronal suspensions and culture conditions. Chick forebrain was dissected from 8-day embryos (E8) and rat septum, hippocampus, and striatum were from 18-day (E18) fetuses. The tissues were incubated for $10 \mathrm{~min}$ at $37^{\circ} \mathrm{C}$ in $\mathrm{Ca}^{2+}, \mathrm{Mg}^{2+}$-free Hanks' balanced salt solution, and then switched to $0.08 \%$ trypsin in the same solution for an additional $30 \mathrm{~min}$. Following two washes with EBM containing $1 \%$ (w/ v) ovalbumin, the tissues were dissociated in albumin-EBM as previously described (Barbin et al., 1984; Varon et al., 1984c). The cell suspension was then diluted (usually 75 - to 100 -fold) to the desired cell density and with the desired medium, to be used for either culture or biochemical experiments. Neuronal suspensions from E8 chick ciliary ganglia (CG8) were obtained as previously described (Skaper et al., 1984).

Costar 96-well (6-mm diameter) plates were coated with polyornithine, followed by exposure to rat schwannoma conditioned medium, known for its polyornithine-binding neurite-promoting activity (PNPF) (Manthrope et al., 1981). After addition to the culture vessel of the basal medium with its supplements (including CNTF for the ciliary ganglionic cultures), the cell suspension was seeded at a density of 1000 cells/well (2000/well for CG8). The cultures were incubated for 
$24 \mathrm{hr}$ at $37^{\circ} \mathrm{C}$ in an atmosphere of $5 \% \mathrm{CO}_{2} / 95 \%$ air, and then fixed with $2 \%$ glutaraldehyde. Surviving neurons were counted directly under phase microscopy, as described previously (Varon et al., 1984c). Data were expressed as either the actual number of cells per culture or as a percentage of the maximum survival obtained in the best culture conditions.

Titration of neuronotrophic activities. The top well of each 12-well column in a 96 -well plate received $100 \mu \mathrm{l}$ of the undiluted medium to be titrated, and the remaining 11 wells of each column received $50 \mu \mathrm{l}$ of N1-supplcmented EBM. Fifty microliters were then transferred from one well to the next (and $50 \mu \mathrm{l}$ from the last well were discarded) to obtain serial 2 -fold dilutions of the starting material. The numbers of neurons surviving after $24-\mathrm{hr}$ incubation were plotted against the corresponding -fold dilutions to construct titration curves (e.g., Figs. 2 and 3). The fold dilution achieving half-maximal support defines the trophic titer of the original sample, in trophic units (TU) per ml of undiluted material (Varon et al., 1981).

Pyruvate determination. The pyruvate content of basal medium or astroglia-conditioned medium was assayed using the technique described by Von Korff (1969). This method measures spectrophotometrically the oxidation of NADH to NAD which accompanies the reduction of pyruvate to lactic acid in the presence of lactate dehydrogenase (LDH). Figure 1 illustrates the standard curve obtained by applying this method to serially diluted aliquots of EBM which had received an initial $10 \mu \mathrm{mol}$ of pyruvate $/ \mathrm{ml}$. Five per cent differences can be reliably recognized in a $100 \mathrm{nmol} / \mathrm{ml}$ range of pyruvate concentrations.

Pyruvate removal. The method consisted of incubating pyruvatecontaining samples with pyruvate dehydrogenase $(\mathrm{PDH})$ in the presence of $\beta \mathrm{NAD}$, coenzyme $\mathrm{A}$, and thiamine pyrophosphate in a potassium phosphate buffer ( $10 \mathrm{mM}, \mathrm{pH} 7.4)$, according to the procedure described by Stanley and Perham (1980). Reagent concentrations were selected on the basis of the expected pyruvate content of the samples, specifically a 10 -fold excess of PDH (50 milliunits $/ \mathrm{ml}$ ) and $1 \times 10^{-4} \mathrm{M}$ concentrations of the three cofactors. At the end of 30 -min incubation at $37^{\circ} \mathrm{C}$, the reaction was stopped with perchloric acid $(6 \%, v / v$, final concentration), the mixture was neutralized to $\mathrm{pH} 7.4$ with $3 \mathrm{M} \mathrm{K}_{2} \mathrm{CO}_{3}$ and centrifuged $\left(1700 \times g, 10 \mathrm{~min}, 4^{\circ} \mathrm{C}\right)$, and the supernatant fraction was collected. In one aliquot, residual pyruvate was measured by the LDH procedure described in the preceding section. Another aliquot of the same mixtures was passed through an Amicon ultrafiltration cell fitted with a PM-10 membrane, and the filtrate fraction (LMW) was assayed for CNS trophic activity using chick forebrain neurons. Reaction mixtures lacking the PDH enzyme were used as additional controls.

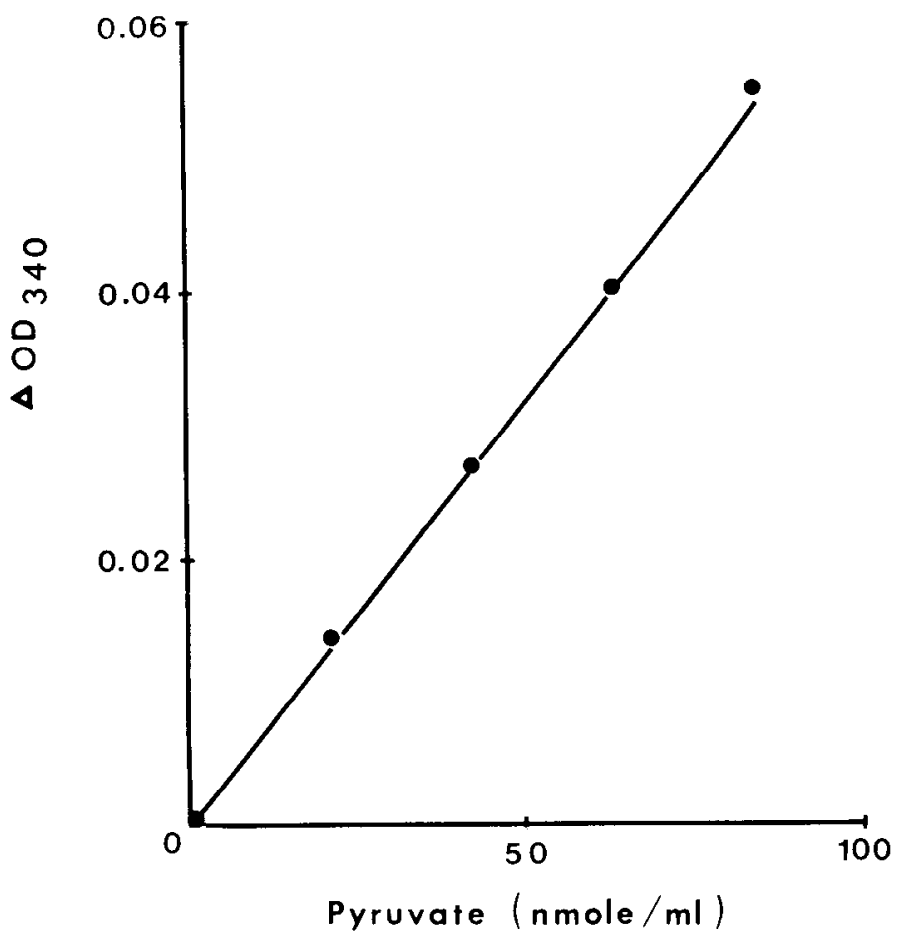

Figure 1. Standard curve for enzymatic titration of pyruvate in EBM solutions. See "Materials and Methods" for experimental details.
Materials. Sources of materials were: EBM and DMEM from Grand Island Biological Company, Grand Island, NY; MEM, F-12, L15, and RPMI-1640 from the Core Culture Facility of the University of California, San Diego; trypsin from Nutritional Biochemicals, Cleveland, $\mathrm{OH}$; penicillin from Parke-Davis, Detroit, MI; bovine crystalline insulin, human transferrin ( $90 \%$ iron-free), progesterone, putrescine dihydrochloride, ovalbumin, pyruvate, serine, glycine, L-glutamine, PDH, $\beta \mathrm{NAD}$, coenzyme $\mathrm{A}$, thiamine pyrophosphate, and polyornithine hydrobromide $\left(M_{\mathrm{r}}=30,000\right)$ from Sigma, St. Louis, MO; sodium selenite from ICN Pharmaceuticals, Plainview, NY; NADH and LDH from Boehringer-Mannheim. The CNTF was a gift from Dr. Manthorpe in this laboratory.

\section{Results}

Comparison of neuronal survival in different serum-free N1supplemented basal media. We have previously demonstrated that EBM preconditioned on astroglial cell cultures (ACM) can support neurons from different chick and rat CNS origins (Varon et al., 1984c). The maximal neuronal survival obtained in the presence of astroglia-conditioned EBM was used here as the reference point for neuronal survivals obtainable in different culture conditions. Table I (upper part) shows the numerical survival of CNS neurons when cultured in different basal media (all serum-free and N1-supplemented). In the presence of EBM (or any other basal medium, data not shown) supplemented with ACM, the maximal neuronal survival was about $60 \%$ of the total number of cells (neurons plus nonneurons) seeded, except for striatal cell cultures where survival was about $40 \%$. In the absence of ACM, the three media EBM, MEM, or RPMI-1640 were not able to support CNS neuronal survival. In contrast, two other media, F-12 and L15, were able to support to some extent survival of all four types of CNS neurons despite the lack of ACM: neurons from E8 chick forebrain (cFB) were supported maximally, while survival of E18 rat CNS neurons was 20 to $30 \%$ lower than that obtained with conditioned medium. Lastly, another medium, DMEM, achieved the maximal neuronal survival obtained with conditioned medium for all four CNS test populations.

Comparisons of chemical compositions of the three supportive media (DMEM, F-12, and L15) with those of the three "incompetent" media (EBM, MEM, and RPMI-1640) defined three types of differences: $(i)$ ingredients present in all media, but considerably higher in the competent ones, (ii) ingredients only present in the competent ones, and (iii) ingredients absent in the competent media (which might, therefore, exert inhibitory influences in the incompetent ones). Figure 2 shows a

\section{TABLE I}

Survival of CNS neurons in different serum-free, N1-supplemented basal media or selected constituents thereof

Test neurons were dissociated from E8 chick forebrain (cFB) and from E18 rat hippocampus (rHP), septum (rSP) or striatum (rSTR), and seeded (1000 cells/well) in 6 -mm wells precoated with PNPFpolyornithine. PYR, pyruvate $\left(1.0 \times 10^{-3} \mathrm{M}\right)$; Ser, serine $\left(4.0 \times 10^{-4}\right.$ $\mathrm{M})$; Gly, glycine $\left(4.0 \times 10^{-4} \mathrm{M}\right) ; \mathrm{Fe}^{3+}, \mathrm{FeCl}_{3}\left(\mathrm{Fe}^{3+}, 2.5 \times 10^{-7} \mathrm{M}\right)$. Data are averaged from duplicate cultures in each of two experiments.

\begin{tabular}{lrrrr}
\hline \multirow{2}{*}{ N1-Containing Media + Supplements } & \multicolumn{4}{c}{ Neurons/Well at 24 hr } \\
\cline { 2 - 5 } & CFB & rHP & rSP & rSTR \\
\hline EBM + ACM (1:1) & 590 & 570 & 570 & 420 \\
EBM or MEM or RPMI-1640 & 0 & 0 & 0 & 0 \\
& & & & \\
F-12 or L15 & 580 & 420 & 410 & 340 \\
DMEM & 620 & 650 & 600 & 400 \\
& & & & \\
EBM + PYR + Ser + Gly + Fe & 660 & 620 & 580 & 410 \\
EBM + + + Ser + Gly + Fe & 0 & 0 & 0 & 0 \\
EBM + PYR & 510 & 580 & 590 & 390 \\
Glucose-free EBM + PYR & 500 & 600 & 560 & 420 \\
\hline
\end{tabular}




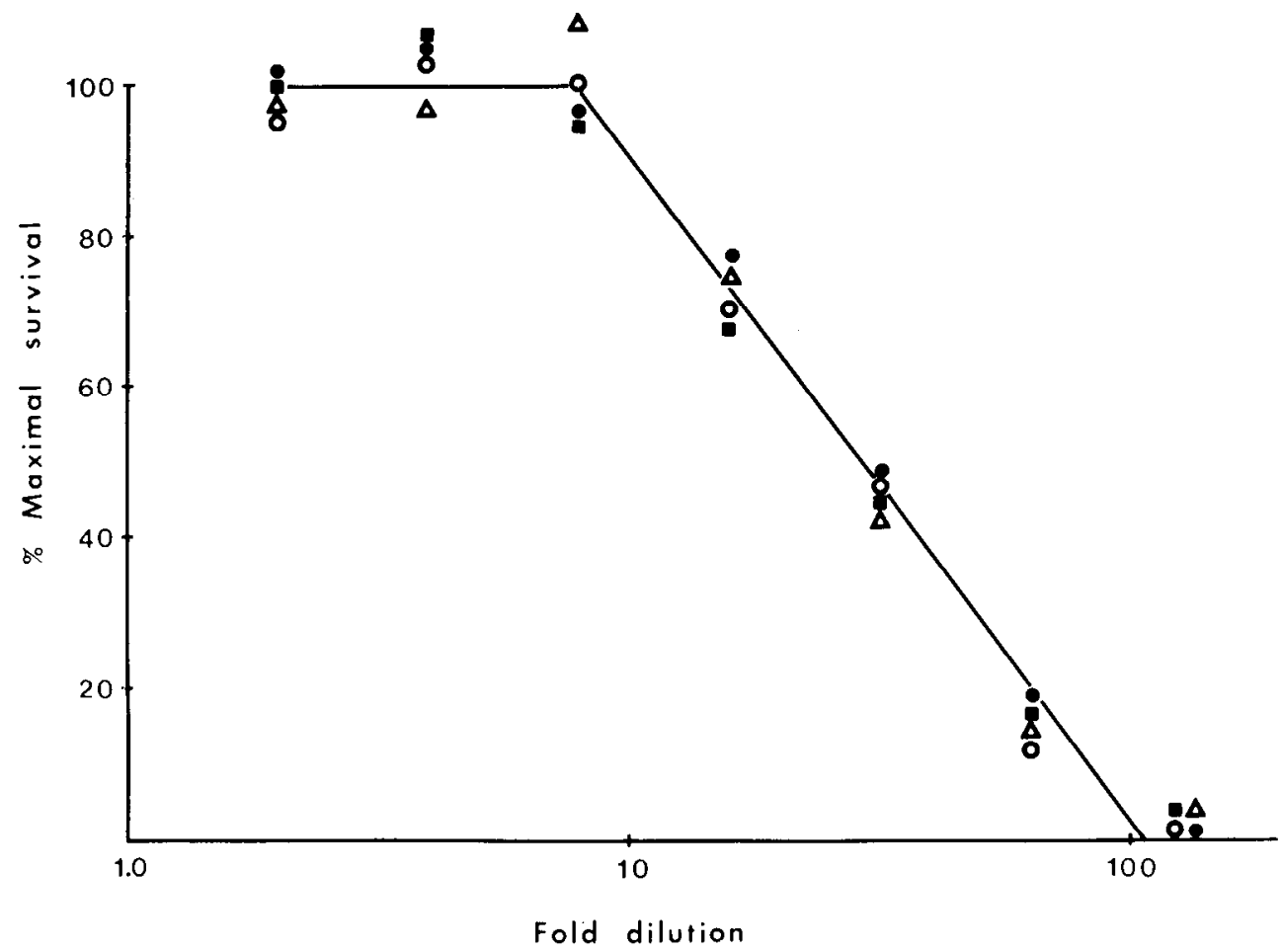

Figure 2. Titration of the survivalsupportive competence of DMEM. Cells derived from $\mathrm{E} 8$ chick forebrain (ם), and E18 rat hippocampus (O), septum $(O)$, and striatum $(\triangle)$ were cultured in $6-\mathrm{mm}$ wells $(1000$ cells/well) with serial dilutions of DMEM in N1supplemented EBM as described under "Materials and Methods." Maximal support for each neuronal population was equated to $100 \%$ at $24 \mathrm{hr}$.

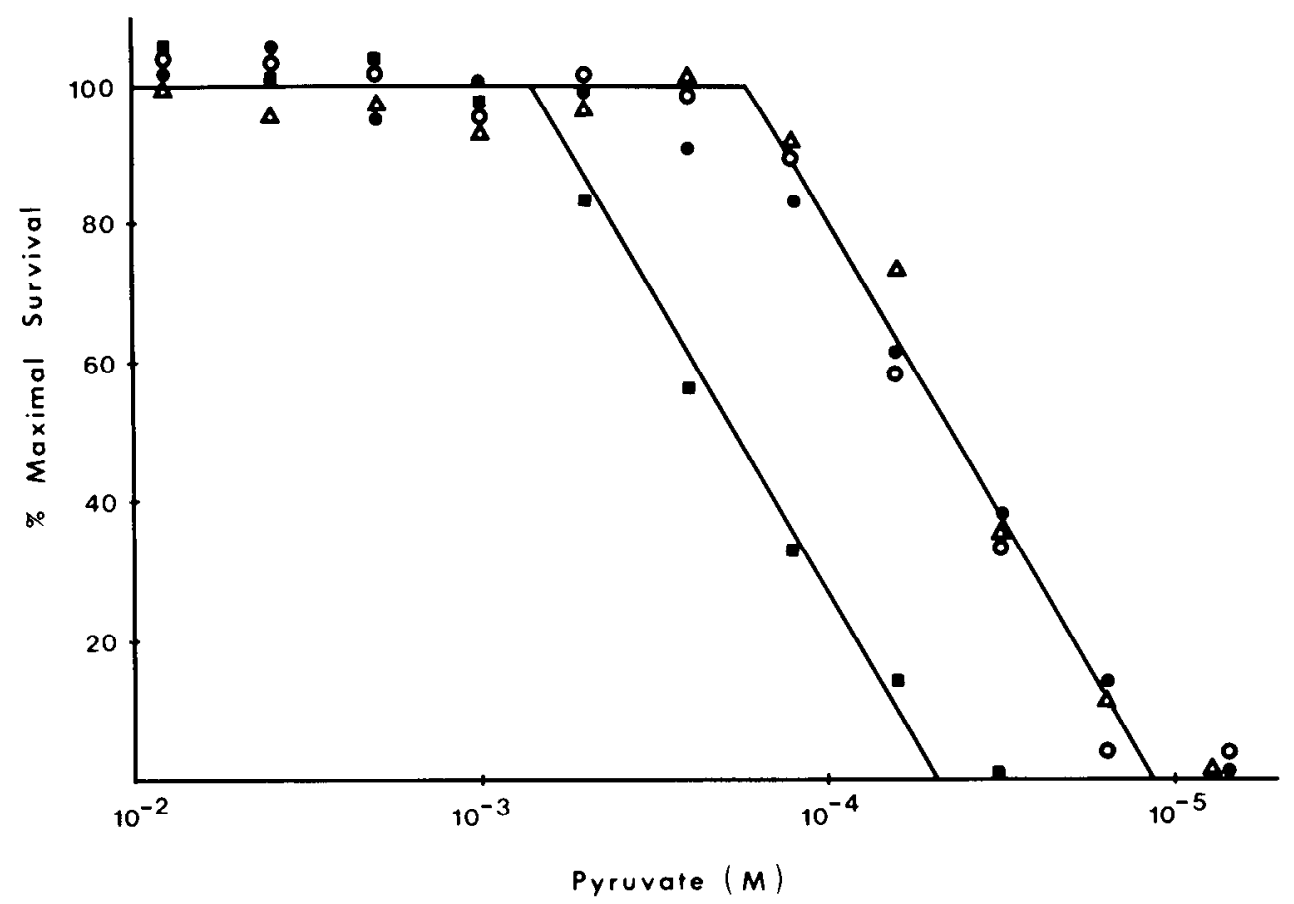

Figure 3. Titration of the pyruvate requirement for survival of CNS neurons. One thousand test cells were seeded into 6-mm wells containing N1supplemented EBM and the indicated concentrations of pyruvate. Neuronal survival at $24 \mathrm{hr}$ is expressed as a percentage of the maximally supported (plateau) level of $100 \%$. Rat hippocampus $(\odot)$, septum $(O)$, striatum $(\triangle)$, and chick forebrain ( $\mathbf{\square}$.

titration of the survival-supportive competence of DMEM used after several dilutions with EBM. For easier comparison, maximal support for each neuronal population was equated to $100 \%$ : as seen in Table I, this corresponded to about 600 neurons/well in all but the rat striatal cultures (about 400 neurons/well). A 5 -fold dilution still permitted full support of CNS neurons, arguing against the relevance of ingredients which are present at 4-fold higher concentrations in DMEM than EBM.

Four DMEM ingredients are entirely missing in EBM: pyruvate, glycine, serine, and ferric ions $\left(\mathrm{Fe}^{3+}\right)$. Table I (lower part) demonstrates that addition of all four such ingredients (at their original DMEM concentrations) conferred on EBM the ability to support optimally the survival of all four types of test neurons. Serine, glycine, and $\mathrm{Fe}^{3+}$ added individually or in combinations had no supportive effects in the absence of pyruvate. Pyruvate alone, on the other hand, when supplied at 1.0 $\times 10^{-3} \mathrm{M}$ (the original DMEM concentration) was sufficient to confer on EBM maximal supportive capability for all three rat CNS populations and near-maximal (about $80 \%$ ) capability for the chick forebrain neurons. Since all basal media contain glucose $\left(5.5\right.$ to $\left.33.3 \times 10^{-3} \mathrm{M}\right)$, we also examined CNS neuronal survival in N1-supplemented EBM from which glucose had been omitted. Even in such a medium, addition of pyruvate resulted in maximal neuronal support, ruling out a concurrent requirement for glucose when pyruvate is available.

Titration of the pyruvate requirements by CNS neurons. 
Pyruvate was added to N1-supplemented EBM at $10 \mathrm{mM}$, a 10 fold excess relative to its DMEM concentration, and the resulting medium was serially diluted with N1-EBM in a titration plate over 4 orders of magnitude. Figure 3 shows the progressive decline in neuronal survival for the four CNS test populations. Maximal survival (equated to $100 \%$ in all cases) was maintained with at least 10 -fold dilutions, to $1 \mathrm{mM}$ pyruvate (henceforth designated as PYR-EBM). As already noted (Table 1), maximal survival in PYR-EBM was the same as in DMFM for the rat neurons, but was only 75 to $80 \%$ for the E8 chick forebrain neurons. Another difference between chick and rat CNS neurons was clearly revealed by the PYR-EBM titration curves of Figure 3. Half-maximal survival was achieved for all rat CNS neurons at about the same 23 -fold dilution (relative to $1 \mathrm{mM}$ pyruvate), but occurred after only 5 -fold dilution for the chick CNS neurons. This finding was in contrast with the performance of chick and rat neurons in DMEM (Fig. 2), where all four populations were half-maximally supported at the same, about 27 -fold dilution.

The above data indicate that DMEM contains ingredients other than pyruvate which are relevant to E8 chick forebrain neurons in two ways: $(i)$ they rescue additional neurons (20 to $25 \%$ of the maximal number of viable neurons), and (ii) they reduce about 5 -fold the overall requirement for pyruvate. Table II shows that the former, but not the latter, contribution by additional DMEM ingredients can be achieved by adding serine $\left(4 \times 10^{-4} \mathrm{M}\right)$ to PYR-EBM. Serine supplementation raised maximal survival to the full DMEM level, even though it supported no neuronal survival in the absence of pyruvate, suggesting that a subset of E8 chick forebrain neurons requires both pyruvate and serine. Serine, however, did not increase the titer for PYR-EBM even in the additional presence of glycine and $\mathrm{Fe}^{3+}$.

TABLE II

Differential effects on $E 8$ chick forebrain neurons by DMEM and $P Y R-E B M$

Both media contain pyruvate at $1 \mathrm{mM}$ and were supplemented with N1. Serine (Ser), glycine (Gly), and ferric ions $\left(\mathrm{Fe}^{3+}\right)$, the other ingredients of DMEM, were lested at the concentrations shown in Table 1.

\begin{tabular}{lcc}
\hline N1-Containing Media + Supplements & Maximal Survival & Trophic Titers \\
\hline & neurons/wel & TU/ml \\
DMEM & 640 & 28 \\
PYR-EBM & 500 & 5 \\
& & \\
PYR-EBM plus Ser + Gly + Fe ${ }^{3+}$ & 640 & 5 \\
PYR-EBM plus Ser & 620 & 5 \\
EBM plus Ser & 0 & \\
\hline
\end{tabular}

TABLE III

Enzymatic determination of pyruvate in astroglia-conditioned and control media, and their relationship to trophic activities of the media for rodent and avian CNS embryonic neurons

\begin{tabular}{lcccc}
\hline & EBM & DMEM & $\begin{array}{c}\text { EBM }+10^{-3} \\
\text { M PYR }\end{array}$ & ACM \\
\hline Pyruvate content (nmol/ml) & 0 & 910 & 960 & 320 \\
Neuronotrophic activity & & & & \\
$\quad(\mathrm{TU} / \mathrm{ml})$ & & & & \\
Rat hippocampus & 0 & 24 & 31 & 11 \\
$\quad$ Septum & 0 & 30 & 25 & 12 \\
$\quad$ Striatum & 0 & 28 & 24 & 9 \\
Chick forebrian & 0 & 28 & 5 & 11 \\
Pyruvate ED S0 $_{\text {(nmol/TU) }}$ & & & & \\
Rat hippocampus & & 38 & 31 & 29 \\
$\quad$ Septum & & 30 & 38 & 27 \\
$\quad$ Striatum & & 32 & 40 & 36 \\
Chick forebrain & & 32 & 192 & 29 \\
\hline
\end{tabular}

Pyruvate and the LMW trophic agents for CNS neurons in astroglia-conditioned medium. The finding that addition of pyruvate confers on unconditioned EBM a similar trophic competence as does conditioning over glial cell cultures (Table I) raises the question whether pyruvate itself might not be the main LMW agent responsible for the trophic activity displayed by conditioned media for CNS neurons (Varon et al., 1984c).

Pyruvate concentrations were directly measured in $(i)$ DMEM, which includes pyruvate at $1000 \mathrm{nmol} / \mathrm{ml}$; (ii) EBM to which $1000 \mathrm{nmol} / \mathrm{ml}$ pyruvate had been added (PYR-EBM); and (iii) EBM previously conditioned over primary rat astroglial cultures (ACM). All three media were also titrated in parallel for their trophic activity on all four CNS neuronal cultures. The results are summarized in Table III. The pyruvate concentrations determined enzymatically in DMEM and PYREBM were close (91 to $96 \%$ ) to their theoretical values. Astrocyte-conditioned mediun contained enzymatically measurable pyruvate, whereas unconditioned EBM had none. The pyruvate content of ACM was found to be about one-third that of DMEM, or $320 \mathrm{nmol} / \mathrm{ml}$. The biological assay of DMEM and PYR-EBM for CNS trophic activity yielded similar titers on the three rat neuronal cultures $(24$ to $30 \mathrm{TU} / \mathrm{ml})$, and revealed the expected 5 -fold lower competence $(5 \mathrm{TU} / \mathrm{ml})$ of PYR-EBM than DMEM on the chick forebrain neurons. ACM displayed about one-third the activity of the two unconditioned media on the rat neurons (9 to $12 \mathrm{TU} / \mathrm{ml}$ ). Unexpectedly, ACM displayed the same activity level (11 TU/ml) also on the chick neurons.

Relationships between pyruvate contents and trophic activities are readily compared using their ratios (nanomoles/TU). Such ratios correspond to the $\mathrm{ED}_{50}$ for pyruvate, since $1 \mathrm{TU} /$ $\mathrm{ml}$ is defined as the concentration of trophic agent(s) required for half-maximal effect. DMEM yielded ratios of 30 to $40 \mathrm{nmol} /$ $\mathrm{TU}$ with the four neuronal targets, i.e., $\mathrm{ED}_{50}$ values of about $3.5 \times 10^{-5} \mathrm{M}$ pyruvate. PYR-EBM yielded similar ratios with rat neurons and a 5 - to 6-fold higher one on chick neurons (an $\mathrm{ED}_{50}$ of about $2 \times 10^{-4} \mathrm{M}$ pyruvate). Astrocyte-conditioned medium yielded $\mathrm{ED}_{50}$ values in the same range as DMEM (about $3 \times 10^{-5} \mathbf{M}$ ), and did so even with chick neurons.

These results indicate that: $(i)$ the pyruvate content acquired by EBM upon its conditioning by astroglial cells (or by direct addition to fresh EBM) accounts fully and precisely for the trophic activity displayed on rat CNS neurons, and (ii) astroglia conditioning confers on EBM five to six times more trophic activity for chick CNS neurons than can be accounted for by its new pyruvate content alone, bringing the pyruvate apparent $\mathrm{ED}_{50}$ down to that of complete (unconditioned) DMEM.

Further verification of the conclusion suggested by the preceding study was sought by treating DMEM, PYR-EBM, and ACM with $\mathrm{PDH}$ (which converts pyruvate to acetyl coenzyme A) and measuring the corresponding losses in trophic activity of the treated media for chick forebrain neurons. The results are shown in Table IV. Processing the enzyme-free controls did not affect either their measurable contents of pyruvate or their neuronotrophic titers. Treatment with the PDH enzyme reduced the pyruvate content by about $87 \%$ in all three media. The resulting losses in neuronotrophic activity were in the same general range ( 81 to $91 \%$ ), although more widespread across the three media. Correspondingly, the pyruvate $\mathrm{ED}_{50}$ values calculated as nanomoles/TU were those expected from chick forebrain neurons for both the starting pyruvate and that lost as a result of the enzymatic treatment. Thus, the trophic activity of astroglia-conditioned medium for chick CNS neurons is entirely dependent on its content of pyruvate, and the additional glia-supplied ingredients have a "sparing" effect on pyruvate concentrations rather than supplementing he pyruvate content with their own trophic activity, a contribution similar to that already recognized for unidentified DMEM constituents (see Table II). 
TABLE IV

Enzymatic degradation of pyruvate in astroglia-conditioned and control media, and corresponding losses in their trophic activity for chick CNS (forebrain) neurons

\begin{tabular}{|c|c|c|c|}
\hline & DMEM & $\begin{array}{c}\mathrm{EBM}+\mathrm{PYR} \\
\left(10^{-3} \mathrm{M}\right) \\
\end{array}$ & $\begin{array}{c}\text { ACM } \\
\text { (LMW Fraction) }\end{array}$ \\
\hline \multicolumn{4}{|l|}{ Pyruvate content $(\mathrm{nmol} / \mathrm{ml})$} \\
\hline Perchlorate only & 922 & 1,030 & 352 \\
\hline $\begin{array}{l}\text { Perchlorate }+ \text { PDH treat- } \\
\text { ment }\end{array}$ & $\underline{117}$ & $\underline{137}$ & $\underline{44}$ \\
\hline Net loss & 875 & 893 & 308 \\
\hline$\%$ loss & 88 & 87 & 87 \\
\hline \multicolumn{4}{|l|}{ CNS trophic activity (TU/ml) } \\
\hline Before perchlorate & 30.5 & 5.0 & 12.0 \\
\hline Perchlorate only & 30.5 & 5.0 & 12.0 \\
\hline $\begin{array}{l}\text { Perchlorate }+ \text { PDH treat- } \\
\text { ment }\end{array}$ & $\underline{5.9}$ & $\underline{0.6}$ & $\underline{1.1}$ \\
\hline Net loss & 24.6 & 4.4 & 11.9 \\
\hline$\%$ loss & 81 & 88 & 91 \\
\hline \multicolumn{4}{|l|}{ Pyruvate $\mathrm{ED}_{50}(\mathrm{nmol} / \mathrm{TU})$} \\
\hline Before degradation & 33 & 206 & 27 \\
\hline Loss caused by degradation & 36 & 203 & 26 \\
\hline
\end{tabular}

LMW components of ACM and their effects on E8 chick CG8 neurons. The CG8 neurons comprise three subsets, all of which require CNTF for their survival (Skaper et al., 1984). When cultured in EBM $(+\mathrm{N} 1+\mathrm{CNTF})$, subset A does not require pyruvate. Subset $\mathrm{B}$ requires pyruvate with approximate $\mathrm{ED}_{50}$ values (nanomoles $/ \mathrm{ml}$ ) of 450 in the absence of serine, 50 in its presence, and 10 in the presence of both serine and $\mathrm{Fe}^{3+}$. Subset $\mathrm{C}$ requires pyruvate $\left(\mathrm{ED}_{50}=10 \mathrm{nmol} / \mathrm{ml}\right.$ or $\left.1 \times 10^{-5} \mathrm{M}\right)$, but also both serine $\left(\mathrm{ED}_{50}=3 \times 10^{-5} \mathrm{M}\right)$ and $\mathrm{Fe}^{3+}\left(\mathrm{ED}_{50}=3 \times\right.$ $10^{-11} \mathrm{M}$ with transferrin, and $1.5 \times 10^{-9} \mathrm{M}$ without it). Each subset contributes about one-third of the total viable population.

The newly acquired knowledge that pyruvate is found in ACM (about $330 \mathrm{nmol} / \mathrm{ml}$; see Tables III and IV) suggests that the LMW fraction of ACM should also have the ability to rescue part or all of subset $\mathrm{B}$ neurons, provided CN'I'F' and N1 are also made available to the CG8 neuronal culture. In addition, a possible presence of serine and $\mathrm{Fe}^{3+}$ in the same $\mathrm{ACM}$ fraction could be detected by a reduced $\mathrm{ED}_{50}$ for pyruvate and the survival of part or all of subset $\mathrm{C}$ neurons as well. To examine such possibilities, fresh EBM received $300 \mathrm{nmol} / \mathrm{ml}$ of pyruvate, while the fraction derived from ACM (ACMLMW) was diluted by $10 \%$, to obtain approximately comparable starting concentrations of pyruvate. The two media were serially diluted with (i) fresh EBM, (ii) EBM plus excess serine $\left(4 \times 10^{-4} \mathrm{M}\right)$, or (iii) EBM plus excess serine $\left(4 \times 10^{-4} \mathrm{M}\right)$ and $\mathrm{Fe}^{3+}\left(2.5 \times 10^{-7} \mathrm{M}\right)$, all three dilution media also containing both CNTF and N1. The resulting titration curves on CG8 neuronal cultures are shown in Figure 4.

Both PYR-EBM (closed symbols) and ACM-LMW (open symbols) gave essentially the same results. At excess dilutions of all combinations, a base line survival of 260 neurons/culture (about $30 \%$ of maximal survival) was obtained, representing the pyruvate-independent subset A typical of the EBM conditions. Both PYR-EBM and ACM-LMW, in the absence of exogenous serine and $\mathrm{Fe}^{3+}$, supported an additional number of neurons, about 170/culture when tested undiluted (approximately $300 \mathrm{nmol} / \mathrm{ml}$ of pyruvate). This pyruvate concentration is below the $\mathrm{ED}_{50}$ for subset $\mathrm{B}$ (approximately $450 \mathrm{nmol} / \mathrm{ml}$ ), and extrapolation of the titration curve (dotted line on the left side of 1-fold dilution) fitted the expected behavior of serinefree, $\mathrm{Fe}^{3+}$-free cultures. When serine-supplemented media were examined, plateau survival was 400 neurons/culture above base line (the equivalent of subset B added by pyruvate), and both trophic titers were raised by about 10 -fold, with the corresponding reduction of the pyruvate $\mathrm{ED}_{50}$ to $40 \mathrm{nmol} / \mathrm{ml}$. Finally, when both serine and $\mathrm{Fe}^{3+}$ were included in the medium diluent, there was a further 5 -fold shift in titer (pyruvate $\mathrm{ED}_{50}$ reduced to $8 \mathrm{nmol} / \mathrm{ml}$ ), with the concurrent rescue of subset $\mathrm{C}$ for a total survival plateau of about 880 neurons/culture. The parallel behaviors of ACM-LMW and PYR-EBM, well within the variations inherent to these bioassay systems, prompt the un-

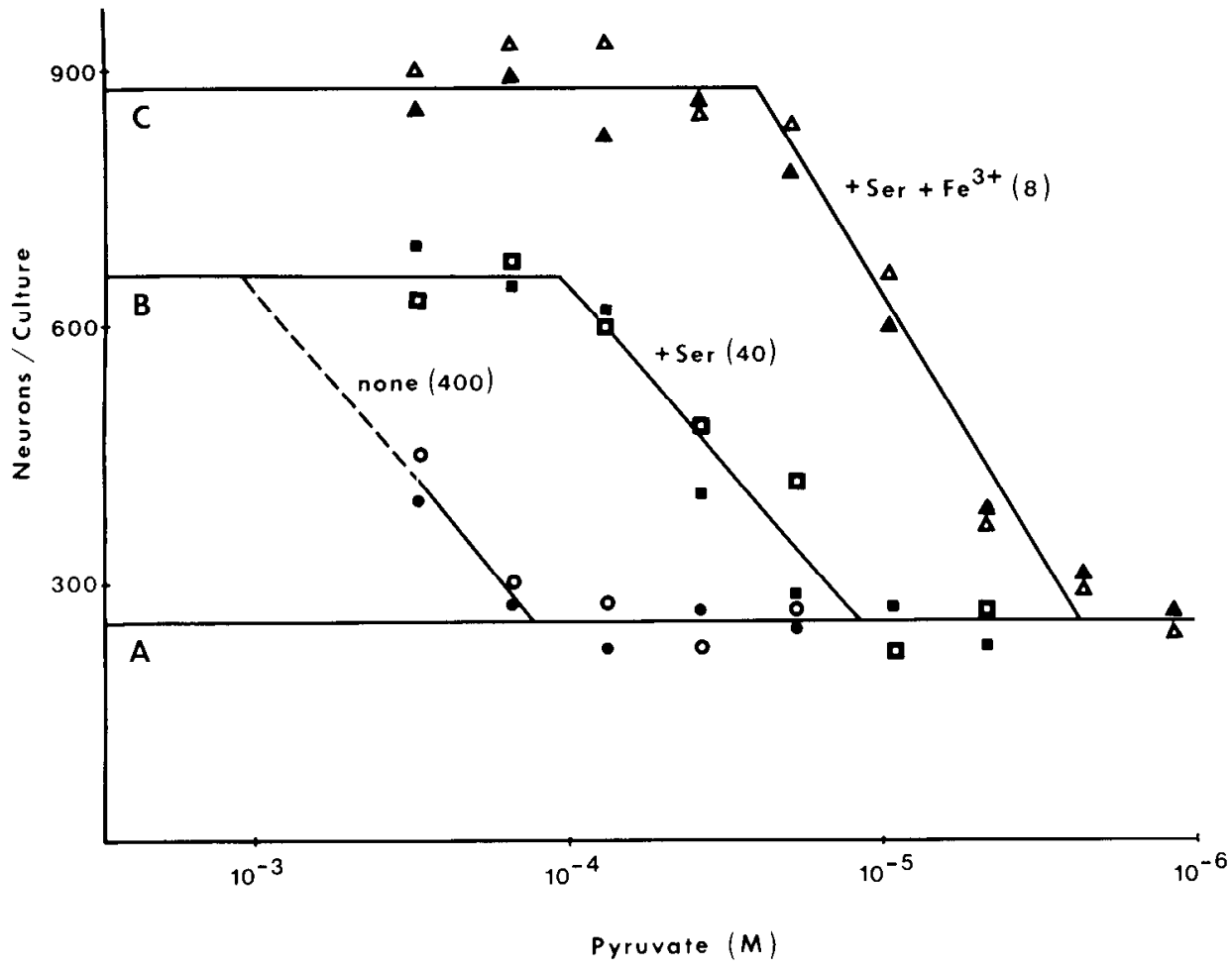

Figure 4. Titration of pyruvate trophic activity for ciliary ganglionic neurons in PYR-EBM (closed symbols) and ACMLMW (open symbols) serially diluted with EBM (+N1 + CNTF) supplemented with no additive or serine or serine plus $\mathrm{Fe}^{3+}$. Numbers in parentheses indicate pyruvate $\mathrm{ED}_{50}$ values. 
equivocal conclusion that the astroglial-conditioned medium tested acquired pyruvate but no demonstrable serine or $\mathrm{Fe}^{3+}$ from the conditioning cells.

\section{Discussion}

In previous studies we had established that glial-conditioned media contain low molecular weight agents which can support survival of CNS neurons in the absence of recognized protein neuronotrophic factors (NTFs), but cannot substitute for NTFs with regard to PNS neurons (Varon et al., 1984c). Independently from those studies, we have found that pyruvate is essential for the survival of about two-thirds of the neurons from E8 chick ciliary ganglia and there, too, the concurrent presence of protein NTFs is required by these PNS neurons (Skaper et al., 1984). The work reported here shows that: (i) primary astroglial cultures release pyruvate into their conditioned medium (ACM); (ii) pyruvate is the critical component of the low molecular weight trophic activity of ACM for both rat and chick CNS neurons; (iii) the pyruvate requirement by chick CNS neurons is reduced by other low molecular weight agents released by the astroglial cells (and/or present in fresh DMEM); (iv) such auxiliary agents are not serine and $F e^{3+}$, as shown by the responses of chick ciliary ganglionic neurons to ACM (in the presence of CNTF).

Intriguing differences are revealed, by these several studies, between chick and rat prenatal CNS neurons and between E8 chick CNS and PNS cells, with regard to $(i)$ the proportion of neurons requiring pyruvate; (ii) additional LMW requirements for neuronal survival, and (iii) auxiliary LMW agents unable to increase neuronal survival but having a sparing effect on the pyruvate concentrations needed. About one-third of chick CG8 neurons survive without exogenous pyruvate, while all the neurons recoverable from these CNS dissociates needed it. Pyruvate-dependent neurons had no other recognized LMW requirement in the case of rat CNS cells, whereas serine and $\mathrm{Fe}^{3+}$ were additionally needed by half the chick CG8 cells and serine by one-quarter the chick forebrain cells. Pyruvate $\mathrm{ED}_{50}$ was low for rat CNS neurons ( 3 to $4 \times 10^{-5} \mathrm{M}$ ) with no assistance from auxiliary "sparing" agents, while similarly low $\mathrm{ED}_{50}$ values were displayed by $\mathrm{E} 8$ chick CG neurons only with the addition of serine and by E8 chick forebrain neurons only through the presence of yet unidentified components of DMEM or astroglia-conditioned medium. Species variations and/or developmental differences might account for such differential behaviors. In any case, further investigations could shed some light on interconnected biochemical pathways which may operate to different degrees in these various neuronal populations.

The inability of glucose-supplemented EBM to support CNS neuronal survival and the converse ability of exogenous pyruvate to support fully survival in the absence of glucose indicate a differential utilization of exogenous glucose and pyruvate by the cell. A defect could lie with glucose transport or metabolism. At present, we have no information on a lack of glucose transport. Glycolytic enzymes do, however, exhibit developmental changes (El-Hassan et al., 1981) which could account for inadequate supplies of glucose-derived intermediary metabolites for embryonic neurons. Pyruvate, being the end product of glycolysis, could exert its survival-supportive action by bypassing a limiting step in the glycolytic pathway.

We would like to speculate that, in vivo, prenatal CNS neurons from diverse origins also depend on an external source of pyruvate (or equivalent intermediate metabolites). The most likely candidate for an external source is the glial cell population. In culture, astroglial cells can utilize glucose for their own support, can synthesize and accumulate glycogen from glucose, and can break glycogen down when challenged by external signals such as $\mathrm{K}^{+}$, norepinephrine, and certain peptides (Magistretti et al., 1983). Cultured astroglial cells can also support neuronal survival directly (Banker, 1980) and generate conditioned media containing low molecular weight constituents capable of supporting neuronal survival in the absence of living glial cells (Varon et al., 1984c). The present findings raise the possibility that conditioned media are trophic for CNS neurons via a pyruvate content acquired from partial glucose utilization by the conditioning glial cell cultures. Direct measurement of pyruvate in astroglia-conditioned medium confirmed a pyruvate output by the glial cells, and the values obained were in good agreement with the relative trophic titers determined using both rat and chick CNS test neurons. Selective removal of pyruvate from ACM resulted in the loss of trophic activity, strengthening the view that no other LMW agents contributed directly to the trophic activity of the medium.

Future investigations may explore the possibility that pyruvate requirements are exacerbated in pathological situations, using in vitro and in vivo models (cf. Varon et al., 1984b). Neuropathological situations in both central and peripheral nervous systems might be based on imposed or enhanced defects in either a neuronal utilization of glucose or pyruvate or a normal supply of pyruvate from extraneuronal sources. Aberrations in pyruvate metabolism and pyruvate-metabolizing enzymes have already been described for several neuropathies (Blass et al., 1976; Kark and Rodriguez-Budelli, 1979).

\section{References}

Banker, G. (1980) Trophic interactions between astroglial cells and hippocampal neurons in culture. Science 209: 809-810.

Barbin, G., I. Selak, M. Manthorpe, and S. Varon (1984) Use of central neuronal cultures for the detection of neuronotrophic agents. Neuroscience 12: 33-43.

Blass, J. P., R. A. Kark, and N. K. Menon (1976) Low activities of the pyruvate and oxoglutarate dehydrogenase complexes in five patients with Friedreich's ataxia. N. Engl. J. Med. 295:62-67.

Bottenstein, J. E., S. D. Skaper, S. Varon, and G. H. Sato (1980) Selective survival of neurons from chick embryo sensory ganglionic dissociates using defined, serum-free supplemented medium. Exp. Cell Res. 125: 183-190.

El-Hassan, A., S. Zubairu, J. S. Hothersall, and A. L. Greenbaum (1981) Age-related changes in enzymes of rat brain. 1. Enzymes of glycolysis, and pentose phosphate pathway and lipogenesis. Enzyme 26: $107-112$.

Kark, R. A., and M. Rodriguez-Budelli (1979) Pyruvate dehydrogenase deficiency in spinocerebellar degenerations. Neurology 29: 126-131.

Magistretti, P. J., M. Manthorpe, F. E. Bloom, and S. Varon (1983) Functional receptors for vasoactive intestinal polypeptide in astroglia cultured from neonatal rat brain. Regul. Peptides 6: 71-80.

Manthorpe, M., S. Varon, and R. Adler (1981) Neurite-promoting factor (NPF) in conditioned medium from RN22 schwannoma cultures: bioassay, fractionation and other properties. J. Neurochem. 37: 759-767.

Manthorpe, M., F. M. Longo, and S. Varon (1982) Comparative features of spinal neuronotrophic factors in fluids collected in vitro and in vivo. J. Neurosci. Res. 8: 241-250.

Skaper, S. D., R. Adler, and S. Varon (1979) A procedure for purifying neuron-like cells in cultures from central nervous tissues with a defined medium. Dev. Neurosci. 2: 233-237.

Skaper, S. D., I. Selak, M. Manthorpe, and S. Varon (1984) Chemically defined requirements for the survival of cultured 8 day chick embryn ciliary ganglionic neurons. Brain Res. 302: 281-290.

Stanley, C. J., and R. N. Perham (1980) Purification of 2-oxo acid dehydrogenase multienzyme complexes from ox heart by a new method. Biochem. J. 191: 147-154.

Varon, S., and R. Adler (1981) Trophic and specifying factors directed to neuronal cells. Adv. Cell. Neurobiol. 2: 115-163.

Varon, S., S. D. Skaper, and M. Manthorpe (1981) Trophic activities for dorsal root and sympathetic ganglionic neurons in media conditioned by Schwann and other peripheral neurons. Dev. Brain Res. 1 . $73-87$

Varon, S., M. Manthorpe, I. Selak, and S. D. Skaper (1984a) Humoral agents modulating neuronal survival in vitro. In Symposia in Neuroscience, G. Biggio, P. F. Spano, G. Toffano, and G. L. Gessa, eds., 3rd Capo Boi Conference on Neuroscience (Sardinia, Italy). Pergamon Press, New York, in press.

Varon, S., M. Manthorpe, and L. R. Williams (1984b) Neuronotrophic and neurite promoting factors and their clinical potentials. Dev. Neurosci. $6: 73-100$

Varon, S., S. D. Skaper, G. Barbin, I. Selak, and M. Manthorpe (1984c) Low molecular weight agents support survival of cultured neurons from the central nervous system. J. Neurosci. 4*654-658.

Von Korff, R. W. (1969) Methods Enzymol. 13: 519-523. 